Bull. Austral. Math. Soc.

VOL. 49 (1994) [81-84]

\title{
PRIMITIVE IDEALS OF THE QUANTISED ENVELOPING ALGEBRA OF A COMPLEX LIE ALGEBRA
}

\section{SEI-QWON OH}

In this paper, we characterise all primitive ideals of the quantised enveloping algebra $U_{q}[s l(2, \mathbb{C})]$ of the complex Lie algebra $s l(2, \mathbb{C})$ and show how they are similar to those of $U[s l(2, \mathbb{C})]$, the enveloping algebra of $s l(2, C)$.

The quantised enveloping algebra $U_{q}[s l(2, \mathbb{C})]$ of the complex Lie algebra $s l(2, \mathbb{C})$ is the algebra $\mathbb{C}\left[E, K, K^{-1}, F\right]$ defined by the relations

$$
K E=q^{2} E K, K F=q^{-2} F K, E F-F E=\frac{K^{2}-K^{-2}}{q^{2}-q^{-2}},
$$

where $q$ is not a root of unity. Therefore $U_{q}[s l(2, \mathbb{C})]$ is the iterated Ore extension $\mathbb{C}[E]\left[K, K^{-1} ; \sigma\right][F ; \tau, \delta]$, where $\sigma(E)=q^{2} E, \tau(E)=E, \tau(K)=q^{2} K, \delta(E)=$ $-\left(K^{2}-K^{-2}\right) /\left(q^{2}-q^{-2}\right), \delta(K)=0$. Hence $U_{q}[s l(2, \mathbb{C})]$ is a noetherian domain and the sets $\Re_{1}=\left\{E^{i} K^{j} F^{n} \mid(i, n) \in \mathbb{Z}^{+} \times \mathbb{Z}^{+}, j \in \mathbb{Z}\right\}$ and $\Re_{2}=\left\{F^{i} K^{j} E^{n} \mid(i, n) \in\right.$ $\left.\mathbb{Z}^{+} \times \mathbb{Z}^{+}, j \in \mathbb{Z}\right\}$ are its $\mathbb{C}$-bases and its Gelfand-Kirillov dimension is 3. The element $\Omega=E F+\left(q^{-2} K^{2}+q^{2} K^{-2}\right) /\left(q^{2}-q^{-2}\right)^{2}=F E+\left(q^{2} K^{2}+q^{-2} K^{-2}\right) /\left(q^{2}-q^{-2}\right)^{2}$ is a central element of $U_{q}[s l(2, \mathbb{C})]$.

The quantised enveloping algebra $U_{q}(\mathfrak{g})$ of a semisimple Lie algebra appears in [2] (originally defined by Drinfield, Jimbo, and others). Moreover the structure of a finite dimensional irreducible module of $U_{q}(g)$ is also shown in [2]. In particular, all finite dimensional irreducible modules of $U_{q}[s l(2, \mathbb{C})]$ are of the form $L\left(\omega q^{m}\right)$, where $\omega \in\{1,-1, i,-i\}, m \in \mathbb{Z}^{+}$, where $L(\lambda)$ is the irreducible highest weight module with highest weight $\lambda \in \mathbb{C}^{*}$. (See Lemma 5 for the definition of $L(\lambda)$.)

The structure of a finite dimensional irreducible module of $U_{q}[s l(2, \mathbb{C})]$ is a parallel property of the (classical) enveloping algebra $U[s l(2, \mathbb{C})]$. It is well known that primitive ideals of $U[s l(2, \mathbb{C})]$ with infinite codimensions are of the form $\left\langle\Omega^{\prime}-c\right\rangle$, where $c \in \mathbb{C}$ and $\Omega^{\prime}$ is Casimir element of the enveloping algebra $U[\operatorname{sl}(2, \mathbb{C})]$. In particular, the ideal $\left\langle\Omega^{\prime}-c\right\rangle$ is maximal if and only if $c \in \mathbb{C}$ is not of the form $n^{2}+2 n, n \in \mathbb{N}$. In this paper, we show the following quantum version:

Received 16th February, 1993

This research was partially supported by non directed research fund, Korea Research Foundation, 1992.

Copyright Clearance Centre, Inc. Serial-fee code: 0004-9729/94 \$A2.00+0.00. 


\section{TheOREM 1.}

(1) All primitive ideals of $U_{q}[s l(2, \mathbb{C})]$ with finite codimensions are the annihilators of $L\left(\omega q^{m}\right)$, where $\omega \in\{1,-1, i,-i\}, m \in \mathbb{Z}^{+}$.

(2) For every $c \in \mathbb{C}$, the ideal $\langle\Omega-c\rangle$ is a primitive ideal of $U_{q}[s l(2, \mathbb{C})]$ with infinite codimension. In fact the ideal $(\Omega-c)$ is the annihilator of $L(\lambda)$ for some $\lambda \in \mathbb{C}^{*}$. Conversely, any primitive ideal with infinite codimension is $\langle\Omega-c\rangle$ for some $c \in \mathbb{C}$. Moreover, the ideal $\langle\Omega-c\rangle$ is maximal if and only if $c \in \mathbb{C}$ is not of the form $\pm\left(q^{2(m+1)}+q^{-2(m+1)}\right) /\left(q^{2}-q^{-2}\right)^{2}$, $m \in \mathbb{Z}^{+}$.

CoRollary 2. The centre of $U_{q}[s l(2, \mathbb{C})]$ is just the subalgebra $\mathbb{C}[\Omega]$.

Proof: It follows immediately from Theorem 1 and 8.4.18 of [3].

Since $\Omega$ is central in $U_{q}[s l(2, \mathbb{C})]$ all primitive ideals contain an element $\Omega-c$ for some $c \in \mathbb{C}$ by 8.4 .18 of [3]. First we prove that the ideal $\langle\Omega-c\rangle$ is completely prime.

Lemma 3. Let $R$ be a domain, $\sigma \in A u t(R)$ and let $\delta$ be a left $\sigma$-derivation. Put $S=R[X ; \sigma, \delta]$ the skew polynomial ring over $R$. If $a X+b \in S$ is a normal element and $a$ is invertible in $R$, then the ideal $S(a X+b)$ is completely prime in $S$. (An element $y \in S$ is said to be normal provided $y S=S y$.)

Proof: Notice that $\operatorname{deg}(f g)=\operatorname{deg}(f)+\operatorname{deg}(g)$ for nonzero $f, g \in S$, and given $f \in$ $S$, there are $h \in S, r \in R$ such that $f=h(a X+b)+r$ because $a$ is invertible. Given $f=h_{1}(a X+b)+r_{1}$ and $g=h_{2}(a X+b)+r_{2}$, if $f g \in S(a X+b)$ then $r_{1} r_{2} \in$ $S(a X+b)$. Thus $r_{1} r_{2}=0$. Since $R$ is a domain, $r_{1}=0$ or $r_{2}=0$. Therefore the ideal $S(a X+b)$ is completely prime in $S$.

Proposition 4. For any $c \in \mathbb{C}$, the ideal $\langle\Omega-c\rangle$ of $U_{q}[s l(2, \mathbb{C})]$ is completely prime.

ProOF: The set $\mathcal{A}=\left\{E^{i} \mid i \in \mathbb{Z}^{+}\right\}$is a left Ore set of the subalgebra $\mathbb{C}\left[E, K, K^{-1}\right]$. Let $R$ be the localisation $\mathcal{A}^{-1} \mathbb{C}\left[E, K, K^{-1}\right]$. Thus $R=\mathbb{C}\left[E, E^{-1}, K, K^{-1}\right]$ is an integral domain. By Lemma 3, the ideal $\langle\Omega-c\rangle$ is completely prime in the skew polynomial ring $R[F ; \sigma, \delta]$ where $\sigma(E)=E, \sigma(K)=q^{2} K, \delta(E)=-\left(K^{2}-K^{-2}\right) /\left(q^{2}-q^{-2}\right)$, $\delta(K)=0$.

We prove that the ideal $\langle\Omega-c\rangle$ is completely prime in $U_{q}[s l(2, \mathbb{C})]$. Given $f, g \in$ $U_{q}[s l(2, \mathbb{C})]$, if $f g \in\langle\Omega-c\rangle$ then $f \in R[F ; \sigma, \delta](\Omega-c)$ or $g \in R[F ; \sigma, \delta]\langle\Omega-c\rangle$, say $f \in R[F ; \sigma, \delta]\langle\Omega-c\rangle$. Hence $E^{t} f=h(\Omega-c)$ for some $t \in \mathbb{Z}^{+}$and $h \notin E U_{q}[s l(2, \mathbb{C})]$. We give an order relation in the basis $\Re_{1}$ as follows:

$$
E^{i} K^{j} F^{n}<E^{i^{\prime}} K^{j^{\prime}} F^{n^{\prime}} \text { if }(i, j, n)<\left(i^{\prime}, j^{\prime}, n^{\prime}\right) \text { in the lexicographic order. }
$$

Express $E^{t} f$ and $h$ as linear combinations of the basis $\Re_{1}$. Since $h \notin E U_{q}[s l(2, \mathbb{C})]$, the set $T=\left\{K^{j} F^{n} \mid\right.$ the coefficient of $K^{j} F^{n}$ in the expression of $h$ is nonzero $\}$ is 
nonempty. Let $K^{r} F^{*}$ be the maximal monomial in the set $T$. Since $\Omega-c$ is central and $K E=q^{2} E K$, we have the following:

$$
\begin{aligned}
h(\Omega-c)= & \left(\alpha K^{r} F^{\prime}+\sum_{(i, j, n) \neq(0, r, a)} \alpha_{i j n} E^{i} K^{j} F^{n}\right)(\Omega-c) \\
= & \alpha K^{r}\left(E F+\frac{q^{-2} K^{2}+q^{2} K^{-2}}{\left(q^{2}-q^{-2}\right)^{2}}-c\right) F^{s} \\
& +\sum_{(i, j, n) \neq(0, r, \alpha)} \alpha_{i j n} E^{i} K^{j}\left(E F+\frac{q^{-2} K^{2}+q^{2} K^{-2}}{\left(q^{2}-q^{-2}\right)^{2}}-c\right) F^{n} \\
= & \alpha q^{-2}\left(q^{2}-q^{-2}\right)^{-2} K^{r+2} F^{\prime}+\text { other terms, }
\end{aligned}
$$

where $\alpha$ is nonzero. Therefore we have $t=0$ and so $f$ is in the ideal $(\Omega-c\rangle$.

DEFinition:

(1) Let $\rho: U_{q}[s l(2, \mathbb{C})] \longrightarrow \operatorname{End}_{\mathbb{C}}(M)$ be a representation and $\lambda \in \mathbb{C}^{*}$. A nonzero vector $v \in M$ is said to be a highest weight vector with weight $\lambda$ if $\rho(K) v=\lambda v, \rho(E) v=0$.

(2) If $M$ is generated by a highest weight vector $v$ with weight $\lambda, M$ is said to be a highest weight module with highest weight $\lambda$.

LEMMA 5.

(1) Every homomorphic image of a highest weight module is also a highest weight module with the same highest weight.

(2) For every $\lambda \in \mathbb{C}^{*}$, there exists an irreducible highest weight module with highest weight $\lambda$, denoted by $L(\lambda)$.

Proof: See Proposition 4 of [2] and its proof.

LEmma 6. Let $\rho: U_{q}[s l(2, \mathbb{C})] \longrightarrow E n d_{\mathbb{C}}(L(\lambda))$ be a representation and $\lambda \in \mathbb{C}^{*}$. The irreducible module $L(\lambda)$ is finite dimensional over $\mathbb{C}$ if and only if $\lambda$ is of the form $\omega q^{m}$, where $\omega \in\{1,-1, i,-i\}, m \in \mathbb{Z}^{+}$.

ProOF: Theorem 1 of [2].

Lemma 7. Let $R$ be a $\mathbb{C}$-algebra and let $M$ be an irreducible $R$-module with $a n_{R} M=P$. Then $P$ is of finite codimension (that is, $\operatorname{dimc} R / P<\infty$ ) if and only if $\operatorname{dim}_{\mathrm{C}} M$ is finite.

Proof: If $P$ is of finite codimension then $\operatorname{dim}_{\mathrm{c}} M$ is finite because $M$ is a finitely generated $R / P$-module. Conversely, let $\operatorname{dim}_{\mathrm{C}} M$ be finite. The kernel of the representation $\rho: R \longrightarrow \operatorname{End}_{\mathbf{c}} M$ is just $P$. Hence $P$ is of finite codimension. 
Proof of Theorem 1: If $J$ is a primitive ideal containing $\langle\Omega-c\rangle$ properly, the Gelfand-Kirillov dimension of $U_{q}[s l(2, \mathbb{C})] / J$ is less than or equal to 1 by $[1,8.3 .6]$. Since there is no nonartinian finitely generated noetherian primitive $\mathbb{C}$-algebra with Gelfand-Kirillov dimension 1 (see the proof of $[4,3.2]$ ), the codimension of $J$ is finite by $[1,8.1 .17]$. Hence every primitive ideal with infinite codimension is of the form $\langle\Omega-c\rangle$. Therefore it is enough to show that there exists $\lambda \in \mathbb{C}^{*}$ such that $\lambda$ is not of the form $\omega q^{m}$ and such that $\Omega$ acts on $L(\lambda)$ by the scalar $c$ by Lemma 5,6 and 7 .

Let $v$ be a highest weight vector of $L(\lambda)$ on which $\Omega$ acts by the scalar $c$. Then we have

$$
c v=\Omega v=\left(F E+\frac{q^{2} K^{2}+q^{-2} K^{-2}}{\left(q^{2}-q^{-2}\right)^{2}}\right) v=\frac{q^{2} \lambda^{2}+q^{-2} \lambda^{-2}}{\left(q^{2}-q^{-2}\right)^{2}} v .
$$

Thus we have the equation

$$
\frac{q^{2} \lambda^{2}+q^{-2} \lambda^{-2}}{\left(q^{2}-q^{-2}\right)^{2}}=c
$$

For every $c \in \mathbb{C}$, it is enough to show that the equation $\left({ }^{*}\right)$ has a root which is not of the form $\omega q^{m}$. If $c \neq \pm\left(q^{2(m+1)}+q^{-2(m+1)}\right) /\left(q^{2}-q^{-2}\right)^{2},\left(^{*}\right)$ has four roots all of which are not of the form $\omega q^{m}$. If $c=\left(q^{2(m+1)}+q^{-2(m+1)}\right) /\left(q^{2}-q^{-2}\right)^{2},\left({ }^{*}\right)$ has roots $\pm q^{-m-2}$. If $c=-\left(q^{2(m+1)}+q^{-2(m+1)}\right) /\left(q^{2}-q^{-2}\right)^{2},\left(^{*}\right)$ has roots $\pm i q^{-m-2}$.

\section{REFERENCES}

[1] J.C. McConnell and J.C. Robson, Noncommutative noetherian rings (John Wiley, 1988).

[2] M. Rosso, 'Finite dimensional representations of the quantum analog of the enveloping algebra of a complex Lie algebra', Commun. Math. Phys 117 (1988), 581-593.

[3] L.H. Rowen, Ring theory II (Academic Press, 1988).

[4] S.P. Smith, 'A class of algebras similar to the enveloping algebra of sl(2, C)', Trans. Amer. Math. Soc.(2) 322 (1990), 285-314.

Department of Mathematics Chungnam National University

Taejon 305-764

South Kores 